\title{
Helping and Cooperation in Children with Autism
}

\author{
Kristin Liebal · Costanza Colombi · Sally J. Rogers · \\ Felix Warneken $\cdot$ Michael Tomasello
}

Published online: 11 August 2007

(C) Springer Science+Business Media, LLC 2007

\begin{abstract}
Helping and cooperation are central to human social life. Here, we report two studies investigating these social behaviors in children with autism and children with developmental delay. In the first study, both groups of children helped the experimenter attain her goals. In the second study, both groups of children cooperated with an adult, but fewer children with autism performed the tasks successfully. When the adult stopped interacting at a certain moment, children with autism produced fewer attempts to re-engage her, possibly indicating that they had not formed a shared goal/shared intentions with her. These results are discussed in terms of the prerequisite cognitive and motivational skills and propensities underlying social behavior.
\end{abstract}

Keywords Helping - Cooperation · Autism ·

Understanding goals $\cdot$ Sharing goals

\section{Introduction}

A defining characteristic of human social life is cooperation. Human beings cooperate with one another in a much wider range of contexts and in much more complex ways than do other primate species (Richerson and Boyd 2005). A recent

K. Liebal $(\bowtie) \cdot$ F. Warneken · M. Tomasello

Department of Developmental and Comparative Psychology, Max Planck Institute for Evolutionary Anthropology, Deutscher Platz 6, 04103 Leipzig, Germany

e-mail: kliebal@eva.mpg.de

C. Colombi · S. J. Rogers

M.I.N.D. Institute, University of California, Davis, Sacramento, CA, USA theoretical proposal suggested that the need to participate with others in cooperative activities with shared goals and shared intentions (shared intentionality) may have been the driving force in human evolution leading to all kinds of supporting social-cognitive skills such as complex mindreading, joint attention, and cooperative communication (Tomasello et al. 2005). Ontogenetically, skills of shared intentionality emerge soon after infants' first birthday. They develop the capacity to understand others' individual goals and intentions. In addition, at around this same age they also develop the skills and motivation to share goals and intentions with others. The ability to share goals and intentions is of crucial importance as it structures the way infants attend jointly to things with other persons and the way they interact with and imitate others who are attempting to show them how to do things (Tomasello et al. 2005).

Helping and Cooperation

Helping behaviors demonstrate that children understand others' goals; one person struggles to achieve a goal and the child spontaneously assists, showing recognition of that person's individual goal as well as a motivation to contribute to goal achievement. It is well known that young children show empathy for other persons and prosocially help them to achieve their goals from fairly early in the preschool years (see Eisenberg and Fabes 1998, for a review). In a recent study, Warneken and Tomasello (2006) found that even 18-month-old infants spontaneously helped an adult when he, for example, dropped an object accidentally (as opposed to threw it away on purpose) or was struggling to open a cabinet (see also Kuhlmeier et al. 2003; Liszkowski et al. 2006). These results demonstrate that even pre- or just linguistic infants: (1) understand the actor's individual goal, and (2) are motivated to help. 
Full-fledged cooperation involves activities with shared goals and shared intentions. Following Bratman (1992), shared cooperative activities have three main features (slightly modified): (1) the cooperating partners are mutually responsive to each other, (2) they have a shared goal, (3) and they mutually support each other in their roles in order to achieve that shared goal. It was previously believed that only older children engage in full-fledged cooperative activities. Ashley and Tomasello (1998) presented 2- and 3-year-old dyads of peers with a clear tube with a toy inside; to get the toy one child had to pull a string to bring it in front of a door at the same time that the other child operated a lever to open the door. Children were over 3 years old before they could coordinate their behavior and attention skillfully and communicate effectively with one another in this difficult task. Brownell and Carriger $(1990,1991)$ presented pairs of young children with a task in which one child had to manipulate a springloaded handle to bring a toy in front of an opening, and the other child had simply to grab it. Only children at 24 months of age and older were able to coordinate their behavior and attention successfully and repeatedly with a peer.

Recently, Warneken et al. (2006) addressed the question whether even younger infants are successful when they cooperate with a more skillful adult partner compared to studies using peer dyads. They presented 18- and 24-month-old infants with different nonverbal cooperative tasks, i.e., successful task mastery did not require verbal communication. They measured the infants' behaviors depending on the pre-programmed behaviors of the adult. The crucial manipulation was that the experimenter stopped carrying out his role at certain moments in the shared activity (see Ross and Lollis 1987, for the original use of this method). The key question in this manipulation were the infants' responses to the interruption: would they attempt to re-engage the adult in the pursuit of their common goal, or simply continue attempting to solve the problem alone? Results showed that virtually all infants at 18 and 24 months engaged in the cooperative tasks, and moreover, all infants produced at least one communicative attempt aimed at re-engaging the adult during the interruptions. These results suggest that even before the second birthday, typically developing infants are capable of forming a shared goal and then coordinating their behavior and attention with an adult in pursuit of this common purpose (Warneken et al. 2006).

Autism

Autism is a neurobiological disorder that is diagnosed by three areas of behavior: (1) impairments in social behavior, (2) deficits in communication and language, and (3) restricted and repetitive behaviors and/or interests (American Psychiatric Association 1994). Autism is present early, with symptoms generally manifest in the first two years of life. While social functioning is severely affected, not all aspects of social behavior are equally impaired in autism. For instance, attachment behavior does not appear to be uniquely impaired in autism, as demonstrated in a series of surprising findings in the 1990s (e.g., Capps et al. 1994; Rogers et al. 1993). Moreover, in social interactions with others, children with autism respond appropriately to social engagement from their parents (Kasari et al. 1993). Furthermore, at least two different groups have shown that children with autism appear to understand other people's intentions regarding actions on objects (Aldridge et al. 2000; Carpenter et al. 2001). These authors based their conclusions on the performance of children with autism in Meltzoff's (1995) Behavioral Re-enactment Procedure, in which the experimenter tried but failed to perform an action on an object. Surprisingly, children with autism, rather than merely copied the precise act of the model, performed his/her intended action. These findings suggest that children with autism are not completely blind to others' minds, but they can "read" the meaning of others' overt behaviors even when it involves intended but unperformed acts on objects. In addition to "reading" intentions regarding actions on objects, children with autism in group studies appear to have some knowledge of what others see (Leekam et al. 1997) or, in some cases, to what others know (Baron-Cohen 1995) regarding objects. Thus, children with autism seem to understand something about other people's actions in terms of individual intentionality, such as their individual perceptions and intentions.

On the other hand, there are striking social impairments that are widely described in the autism literature and that might as well be crucial skills in order to cooperate with others. The imitation deficit is particularly well documented in autism (e.g., Charman et al. 1997; Rogers and Pennington 1991; Rogers 1999; Sigman and Ungerer 1984; Smith and Bryson 1998; Stone et al. 1997; Williams et al. 2004). Because of the pivotal role of imitation in the development of more mature socio-emotional skills (e.g., Meltzoff 1990; Rogers 1999; Rogers and Pennington 1991; Stern 1985) imitative skills are now studied intensively with the aim to understand their role in autism (see Williams et al. 2004). A second well documented area of impairment is impairment in use of joint attention behavior (Bono et al. 2004; Kasari et al. 1990; Leekam et al. 2000; Mundy et al. 1986; Sigman and Mundy 1989; Sigman and Ungerer 1984; Sigman et al. 1986). Children with autism show reduced frequency of initiating bids for joint attention with others by declaratively pointing to or showing objects (e.g., Baron-Cohen 1989a; Charman et al. 1997; Mundy and Willoughby 1996) and responding to others' bids for 
joint attention (e.g., Leekam et al. 1997). Furthermore, several studies have shown that young children with autism, despite their ability to use gestures to request objects, tend not to use gestures to share interest in objects (Baron-Cohen 1989b; Charman et al. 1997; Mundy et al. 1986, 1993). This pattern of findings suggests that children with autism are not necessarily avoidant or unresponsive to social contact, but rather that there is reduced attention to others (Dawson et al. 2004), reduced interpersonal "resonance" with others (Rogers and Pennington 1991), or reduced intrinsic reinforcement from shared interactions with others (Dawson et al. 2004).

Only a few studies have focused on social behaviors like helping and cooperation in children with autism. Concerning helping, Sigman and Ruskin (1999) included a measure of helping in a longitudinal study of people with autism. For example, when an experimenter could not perform an act because his/her hands were occupied, the 10- to 13-year-old participants with autism less frequently assisted the partner to reach his/her goal than children with Down syndrome. Regarding cooperation, Jahr et al. (2000) conducted an intervention study involving cooperative play in six children with autism aged 4-12 years. They used materials that were familiar to the children from school and activities of the kind of one partner builds a fence and the other partner puts a toy animal inside the fence. They instructed the participants to either repeat cooperative play episodes they had observed two models acting out or to verbally describe what they observed first before they repeated themselves. Training was conducted until a certain criterion of correct imitation of the models in the imitation condition or correct verbal description and imitation in the verbal condition were achieved. In the following test Jahr et al. measured units of consecutive play between the child and the partner. They found that none of the children achieved the criterion during a baseline before the training and that they did so only after training with verbal description. After this kind of training, they maintained the increased performance in the test with novel settings and novel partners and during follow-up after 1 month. An open question remains whether the activities reflect fullfledged cooperation with two partners having shared goals and shared intentions. To address this question, in our cooperation study we included interruption periods to investigate children's understanding of the partner's role within a cooperative activity when interaction breaks down. Another study by Downs (2003) surprisingly found that 5- to 9-year-old high functioning children with autism cooperated similarly to a group of typically developing children in a Prisoner's Dilemma task. The author discusses the finding in terms of the suitability of the task as the children interacted with an imaginary friend. However, in addition he promisingly argues that the children received intensive behavioral treatment which might have improved their cooperative skills. To conclude, the reported studies addressed helping and cooperative activities in older children with autism. Our studies aimed to investigate these behaviors in younger children with autism, independent from language.

To summarize, the current studies aimed to investigate social behaviors in children with autism compared to children with other developmental delay. To assess helping (Study 1), we adapted a subset of tasks from Warneken and Tomasello (2006), in which an adult is struggling to grasp out-of-reach objects. Based on the theoretical proposal by Tomasello et al. (2005) and findings of intact intention reading in children with autism, we predicted that children with autism might show no deficits in helping behaviors that involve an understanding of other people's individual goals and the motivation to assist. To assess cooperative behavior (Study 2), we adapted the tasks from Warneken et al. (2006). Children interacted with an adult partner who stopped carrying out her role during predetermined interruption periods. In this manipulation we were particularly interested to see if children tried to re-engage the partner, which would indicate that they understood the partner's role in the joint activity. Based on the theoretical account by Tomasello et al. and well-known deficits of imitation and joint attention, we predicted that shared cooperative activities would be a challenge for children with autism, as they require coordination of attention among self, partner, and task (joint attention abilities) and the formation of shared goals and intentions (plans of action) with the partner.

Because the tasks do not rely on receptive and expressive language, they appeared particularly appropriate for testing young children with autism, given the language difficulties they typically demonstrate. This allowed us to differentiate helpful and cooperative behavior from the verbal impairment generally identified in autism.

\section{Study 1: Helping}

\section{Method}

This study was conducted with the approval of the Human Subjects Committee of the University of California, Davis. Consent forms were reviewed with each family and all questions were answered before consent was obtained and before any measures were gathered. Participants were seen in Rogers' Early Development Lab at the M.I.N.D. Institute. Studies 1 and 2 were conducted within one visit. After a warm-up phase in a play area, the child and her parent shifted to the test room. One parent stayed with the child at all times and was encouraged to intervene or stop 
the session if she felt that the child was uncomfortable. The whole session lasted 60-90 minutes and was videotaped through two-way mirrors.

\section{Participants}

Thirty children were included in this study and comprised two groups: Autism Spectrum Disorder (14 with Autistic Disorder and 1 with Pervasive Developmental Disorder, Not Otherwise Specified [this child was included in the analysis presented as his performance in the experimental tasks did not change the overall group score]) and Developmental Delay of mixed etiology (DD; $n=15$ ). The mixed group of developmentally delayed children was included as a comparison, as has been the practice in previous comparative studies. The heterogeneity of autism supports the use of a heterogeneous comparison group. All of the children were between the ages of 24 and 60 months and were recruited from the participant pool of the M.I.N.D. Institute (UC Davis Medical Center, Sacramento CA). Table 1 presents descriptive and matching information.

There were no significant differences between the children with autism and those with mixed DD on chronological age and nonverbal mental age. A nonverbal developmental score for each child was constructed by averaging together the fine motor and visual reception scores of the Mullen Scales of Early Learning (MSEL) (Mullen 1995). Participants were matched on nonverbal developmental age, which appeared an appropriate matching strategy since the experimental tasks were all nonverbal tasks. The children with autism were free from any other medical condition, had no visual or hearing impairment, walked by 15 months of age, had a nonverbal developmental level of 15 months or higher, spoke English as their first language, had been diagnosed with autism by an outside agency, received current clinical diagnoses of autism by expert researchers in the lab, and met criteria for autism on two diagnostic systems: DSM-IV and Autism Diagnostic Observation Schedule-Generic (ADOS-G) (Lord et al. 1999). The ADOS-G was administered by licensed clinicians or by expert researchers in the lab. All were trained to reliability by a researcher who was trained and reliable with Dr. Catherine Lord's research group. ADOS-G administrators maintained $85 \%$ or better item agreement on three consecutive administrations. The children with autism were receiving 5-40 hours per week of behavioral therapy, $1-2$ hours per week of speech therapy, and 1-2 hours per week of occupational therapy.

Within the DD group, there were four children with speech and language delay, one child with Down syndrome, one child with other genetic abnormalities, and nine children with developmental delays of unknown etiology. The children with DD all had normal vision and hearing, were mobile, had a nonverbal developmental level of 14 months or higher, and spoke English as their first language. None met DSM-IV nor ADOS-G (same administration procedure of the autism group was applied) criteria for autism. The children with DD were receiving 1- 2 hours of speech therapy and 1-3 hours of occupational therapy or physical therapy, as well as special education services.

Six additional children (four autism group, two DD group) had to be excluded from the study due to distress (one child), unavailability for further developmental assessment (one child), videotaping problems (two children), and matching the samples on nonverbal mental age (two children).

Table 1 Participant information sample (Studies 1 and 2)

\begin{tabular}{|c|c|c|c|c|}
\hline & \multicolumn{2}{|c|}{ Autism } & \multicolumn{2}{|c|}{ DD } \\
\hline & Mean $(S D)$ & Range & Mean $(S D)$ & Range \\
\hline $\mathrm{CA}^{\mathrm{a}}$ & $40.3(9.5)$ & $24.0-57.0$ & $43.0(10.1)$ & $28.0-60.0$ \\
\hline Overall $\mathrm{MA}^{\mathrm{a}}$ & $24.2(10.2)$ & $12.8-46.6$ & $31.5(11.5)$ & $11.5-52.8$ \\
\hline NVMA $^{\mathrm{a}}$ & $27.4(9.6)$ & $15.5-53.0$ & $33.4(11.1)$ & $14.5-54.0$ \\
\hline $\mathrm{VMA}^{\mathrm{a}}$ & $21.3(11.8)$ & $10.0-44.0$ & $28.4(11.0)$ & $8.5-51.5$ \\
\hline Family income & $\$ 25,000-\$ 50,000$ & $\$ 25,000-\$ 125,000$ & $\$ 25,000-\$ 50,000$ & $<\$ 25,000-125,000+$ \\
\hline Gender & \multicolumn{2}{|c|}{14 male, 1 female } & \multicolumn{2}{|c|}{12 male, 3 female } \\
\hline Ethnicity & \multicolumn{2}{|c|}{12 Caucasian, 2 Hispanic, 1 African-American } & \multicolumn{2}{|c|}{10 Caucasian, 3 Hispanic, 2 Asian } \\
\hline ADOS-G criteria & \multicolumn{2}{|c|}{15 met criteria } & \multicolumn{2}{|c|}{0 met criteria } \\
\hline Severity of autism ${ }^{\mathrm{b}}$ & $15.25(3.74)$ & $9-22^{\mathrm{c}}$ & $2.27(1.10)$ & $1-4$ \\
\hline
\end{tabular}

${ }^{a}$ Groups showed no statistical differences; ${ }^{b}$ Severity of Autism is based on ADOS-G score (autism spectrum cut-off $=7$; autism cut-off $=10$ ); ${ }^{\mathrm{c}} 9$ was the score obtained by the child with PDD-NOS, the lowest score of children with autism was 11 


\section{Materials and Design}

In experimental conditions, the experimenter (E) accidentally dropped an object. She effortfully reached for it but was unable to retrieve the out-of-reach object. In control conditions, E tossed the object away on purpose and did not reach for it. There were four tasks.

Pen In the experimental condition, E was drawing something with a pen on paper while sitting at a table. She then accidentally dropped the pen to the floor, and reached down effortfully for it from her seat. In the control condition, $\mathrm{E}$ was not drawing and simply threw the pen to the floor purposefully and did not reach for it.

Paper Balls In the experimental condition, E showed the child tongs and a box half filled with paper balls, all at a table. She put another four paper balls on the table, two close to her, two out of reach (close to the child). She started putting the paper balls into the box with help of the tongs, but could not reach the two close to the child even reaching for them effortfully. In the control condition, no box was present, but the paper balls were arrayed as in the experimental condition. E simply picked up the close-by paper balls with tongs and put them back on the table, with no reaching for the others.

Cap In the experimental condition, E put a cap on her head once while sitting at a table. When she was about to repeat this action, she accidentally dropped the cap to the floor, and reached down effortfully for it from her seat. In the control condition, E threw the cap to the floor purposefully and did not reach for it.

Clothespins In the experimental condition, E demonstrated to the child hanging washcloths on a line with clothespins. When she was about to put up the third cloth, she accidentally dropped a clothespin to the floor and was unable to reach it. In the control condition, no line and cloths were present, but E simply threw a clothespin to the floor purposefully and did not reach for it.

Each child received two of these tasks in the experimental condition and two of them in the control condition (with one trial per task), with order of conditions counterbalanced across children. The tasks were presented in two blocks of two between two of the cooperation tasks (elevator task, double-tubes task; see below) - with each block containing one experimental and one control task (same order in both for a given child). In each block, either the pen task or the cap task (because they were so similar) were randomly paired with either the paper-balls task or clothespins task, and assignment of tasks to conditions was counterbalanced across children.

\section{Procedure}

Before each trial we made sure that the child watched. The basic behavior of $\mathrm{E}$ was the same for all tasks. In the experimental condition, after she accidentally dropped the object, E reached for the object for a maximum of 30 seconds, starting of solely focusing on the object and vocalizing her effort to retrieve it (1- 10 seconds), then additionally alternating gaze between the child and the object (11-20 seconds), and, if the child still has not passed it to her, verbalizing her desire for the object (e.g., "Oh, my pen!", 21-30 seconds). E never directly asked for help and verbalization was not considered an instruction, but rather an additional affective marker of the E's intent. A control condition was conducted to rule out the possibility that the mere falling down of the object that is related to the experimenter would elicit picking it up and passing it back to $\mathrm{E}$ although she had not expressed the goal to obtain that object. In this control condition, E purposefully dropped the object and waited with a neutral facial expression for 20 seconds. All participants saw the target object fall (placed out of reach for the paperballs task).

\section{Coding}

All sessions were videotaped and coded from DVD. For each trial we scored whether the children helped, i.e., picked the object up and passed it to E1.

\section{Reliability}

Regarding the scoring of whether they passed the object to $\mathrm{E}$, the first and second authors independently coded $100 \%$ of the data. Interrater agreement was $\kappa=0.84$. The 18 cases of disagreements (among 111 cases) were resolved by discussion.

\section{Results}

Hypotheses were tested two-tailed as we predicted no differences between groups. For some children not all four trials could be administered due to practical reasons ( $M=3.5$ for the autism group, $M=3.9$ for the DD group). Therefore, individual mean proportions (the number of trials with helping, divided by the number of trials administered) were calculated for each condition. This measure is depicted in Fig. 1. A repeated measures 


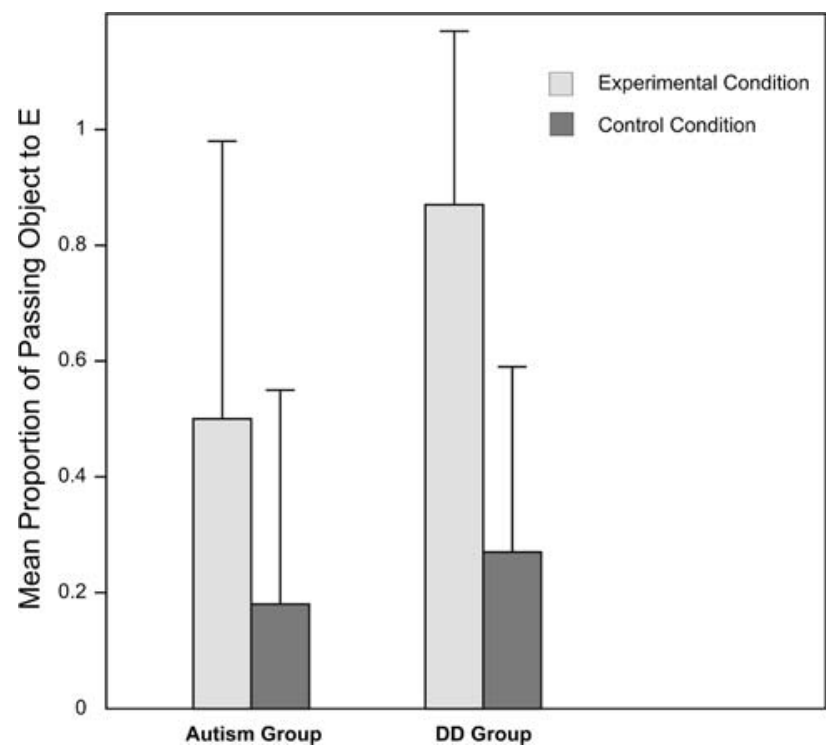

Fig. 1 Mean proportions and standard deviations of helping behavior as a function of group and condition (Study 1)

ANOVA was conducted with Group and Condition as factors. Results revealed a trend for group such that children with developmental delay passed the object to the experimenter more in both conditions than children with autism $(F(1,27)=4.03, p=0.06)$. In addition, a significant main effect for condition was found such that all children helped more in the experimental than in the control condition $(F(1,27)=33.85, p<0.01)$. Importantly, each group independently helped more frequently in the experimental than in the control condition (paired-samples $t$-test: DD: $t(14)=5.39, p<0.01$; autism: $t(13)=2.86, p<0.02)$.

On an individual level, three children with autism (20\%) helped exclusively in the experimental condition, whereas seven children with developmental delay (47\%) did so. Seven children $(47 \%)$ with autism and one child with developmental delay (7\%) never helped. Although there was some small variability among tasks, in neither group was children's performance markedly different on the four tasks.

\section{Discussion}

Both groups of children helped the adult as needed. This required them to understand the adult's goal of obtaining the object and to be motivated to help her. Similarly, Warneken and Tomasello (2006) reported that typically developing children of 18 months of age performed comparably on these tasks. The results of the present study fit well with other reports that children with autism understand something about other persons' goals regarding actions on objects (Aldridge et al. 2000; Carpenter et al.
2001). Thus, not only can they understand another person's goal of action, they also seem to have the motivation to help them with that goal.

However, all of the tasks involved the same type of goal and the same type of helping act, namely, handing over an object to which the other was reaching. It would be interesting to probe their flexibility in helping by including different types of helping situations such as holding the door open for others or completing an unfinished action (e.g., the more complex tasks from Warneken and Tomasello (2006) which we could not administer due to practical and time constraints). Such tasks with more complex goals would also be more similar to the tasks used by Sigman and Ruskin (1999) who, in contrast to the current study, found that children with autism had more difficulties with helping tasks than children with Down syndrome. Thus, it is possible that children with autism can help in situations with simple goals (such as in the current study), but differences become apparent when it concerns more complex goals (such as in Sigman and Ruskin 1999; and some of the tasks in Warneken and Tomasello 2006). Moreover, with regard to the underlying motivation it would be interesting to include situations in which the child herself might have a conflicting selfish motive, or would have to go to some considerable effort to help others.

Helping involves an understanding of another individual's goal and the motivation to assist that person to achieve it. Full-fledged cooperation, on the other hand, involves two partners having a shared goal and shared intentions, relying on one another to perform their respective roles and coordinating their actions and attention in order to achieve their joint goal, which is the focus of the second study.

\section{Study 2: Cooperation}

Method

\section{Participants}

Same as in Study 1.

\section{Materials and Design}

Four tasks were developed (see Fig. 2). Successful task performance required both partners to perform their roles in a coordinated manner.

Tube-with-handles Task The apparatus was a long tube consisting of two parts that could be pulled apart, with a 
Fig. 2 Apparatuses (Study 2)
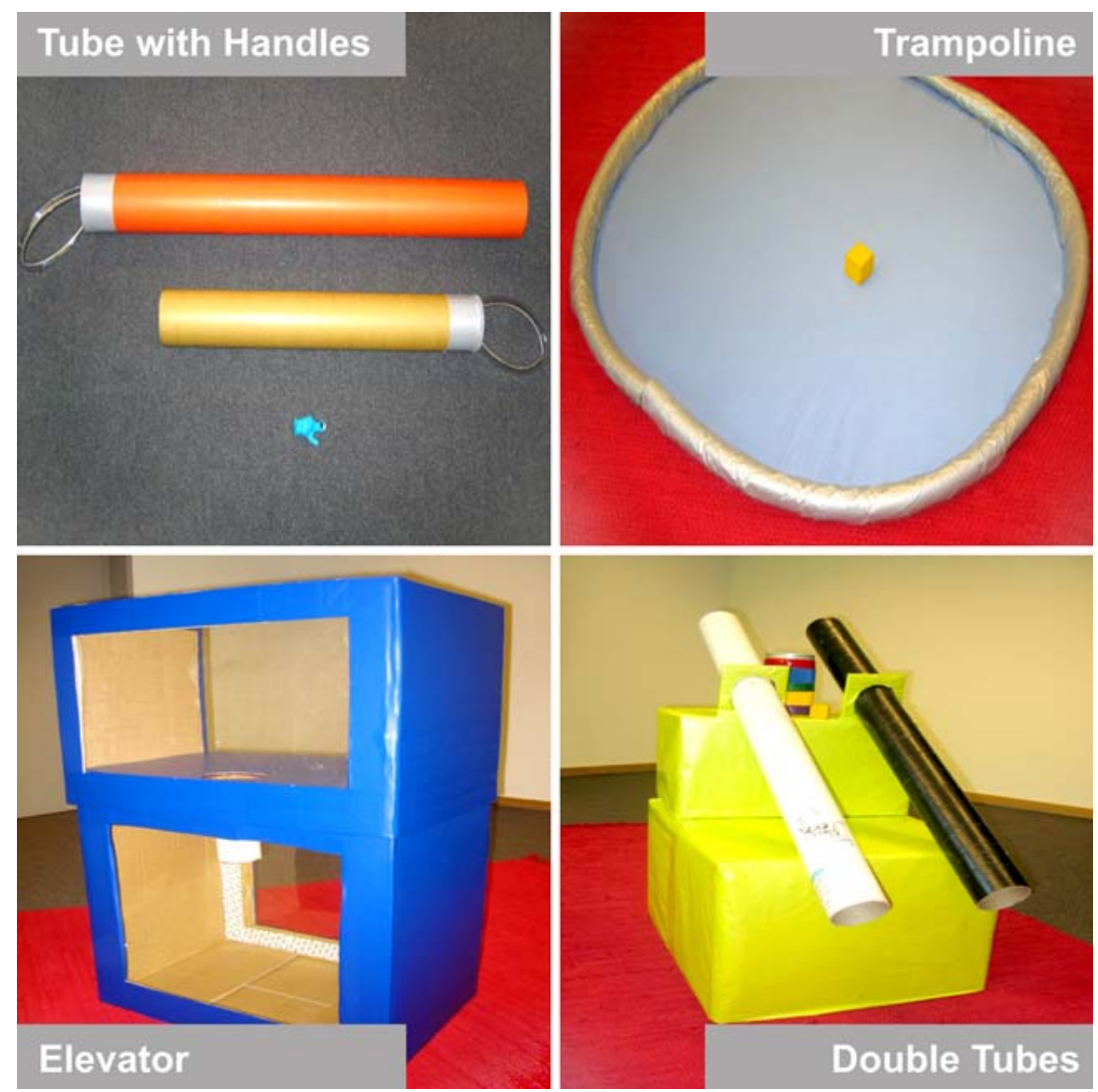

handle at each end. A toy was inside. In order to perform the task successfully and retrieve the toy both partners were required to pull simultaneously at each end. The length of the tube made it impossible for the child to open the tube individually.

Elevator Task The apparatus was a box with a cylinder in which a toy was placed. In order to perform the task successfully and retrieve the toy one partner had to push the cylinder up and hold it in place (role $B$ ) to make the toy accessible for the other partner through a small window in the cylinder on the other side of the apparatus (role A). The size of the box and clear plexiglass screens prevented a single individual from both pushing up the cylinder and grasping the toy inside.

Trampoline Task The apparatus was a big, flexible hoop covered with cloth and a small wooden block. In order to perform the task successfully both partners had to grasp the rim of the hoop-trampoline and make the block bounce by moving the trampoline up and down for a total of $5 \mathrm{sec}$ onds. The size of the apparatus and the construction - the rim was built in a way that it folded if one partner tried to manipulate it individually - prevented the game from being played individually.

Double-tubes Task The apparatus was a stand with two parallel equally long tubes on top, one black and one white, slanting downwards - through which one person could send a small wooden block (role $A$ ), with the other catching it with a tin can at the other end, which made a rattling noise (role $B$ ). In order to perform the task successfully both partners had to choose the same tube to throw down and catch the block at least once for each tube.

Forming a goal involves motivation to achieve it. In order to maximize motivation, we used child-preferred toys for children who did not show interest in the task as described. We had a set of extremely attractive toys which children could select. Additionally, the families were also encouraged to bring their child's favorite small toys which were used as goals in some tasks if needed. Finally, small bits of a favorite food were used in some cases when toys were not attractive to the children. During the test session the parent was asked to not respond to the child's overtures, but to draw the child's attention back to E's activity.

Task order of the four tasks was counterbalanced across children within experimental groups by means of a 
Latin-square design. In the elevator task and double-tubes task, the two roles were administered as a block, with roles counterbalanced within those. Between the two roles of the elevator task and double-tubes task, the two helping tasks were administered - at least partly to distract the child from the role previously performed.

\section{Procedure}

The general procedure for all tasks was as follows: After a short familiarization, the demonstration phase began during which the two female experimenters (E1 and E2) gave a task demonstration with much pleasurable affect. Afterwards, E2 withdrew and E1 initiated trial 1 by starting to perform her role encouraging the child to join by saying "your turn!". If the child was not successful, a second, and, if needed, third demonstration were given. If the child did not perform successfully after demonstration 3 , the task was stopped. If the child succeeded, two interruption trials (called trials 2 and 3) were administered. Each of these trials began with E1 inviting the child's participation and then, after child and E1 had started to engage in cooperative activity, came an interruption period of 15 seconds. During the interruption period, E temporarily stopped interacting, looking at the child with a neutral facial expression. After 15 seconds of interruption, she resumed her role. More details about procedures for each of the four tasks are provided in Appendix 1.

\section{Coding}

All data were coded from DVD by the first author who was partially blind to the diagnoses. For the general performance, we analyzed the percentage of children who passed a task (criteria for successful task performance are described in the task descriptions above).

For the 15-second-interruption periods, we scored children's overall behavior (disengagement, individual attempt, partner-orientation). For each interruption period we coded the behavior every child produced for the majority of time (one code per trial). In addition, we coded the frequency of different communicative attempts during each interruption period. For details, see Appendix 2. To address the relation between helping and cooperative behaviors, a Spearman's rank correlation was calculated.

\section{Reliability}

A random sample of seven subjects (23.3\%) was independently coded by two raters. Cohen's Kappa was computed to measure interrater reliability (Cohen 1960) regarding the behaviors during interruption periods $(\kappa=0.74)$. Regarding the frequency of communicative attempts, weighted Kappa (Sprent and Smeeton 2001) was computed. Interrater agreement was $\kappa=0.88$ for overall communicative attempts, $\kappa=0.73$ for proximal requesting communicative attempts, $\kappa=0.92$ for distal requesting communicative attempts, $\kappa=0.91$ for distal requesting communicative attempts with eye contact, and $\kappa=0.65$ for distal requesting communicative attempts without eye contact.

\section{Results}

Hypotheses were tested one-tailed as we had a directed hypothesis. Results were directed at two major questions addressing general cooperative performance and interruption periods, and finding associations between helping and cooperation by correlating results from Studies 1 and 2 .

\section{General Performance}

Percentage of Children Who Performed a Task We analyzed the percentage of children who successfully passed a task in trial 1 after one to three demonstrations (for criteria of success, see Appendix 1). Significantly fewer children in the autism group than in the DD group passed the tube-with-handles task [autism: 67\%, DD: $100 \%$; Fisher's exact test $(N=30), p<0.02]$, the trampoline task [autism: $60 \%$, DD: $100 \%$; Fisher's exact test $(N=30), p<0.01]$ and the double-tubes-task in both roles [throw: autism: 60\%, DD: 100\%; Fisher's exact test $(N=28), p<0.01$; catch: autism: 33\%, DD: 86\%; Fisher's exact test $(N=29), p<0.01]$. There were no group differences for the elevator task in either role (retrieve: autism: 93\%, DD: 100\%; Fisher's exact test $(N=29)$, $p=0.52$; push: autism: 100\%; DD: 93\%; Fisher's exact test $(N=29), p=0.48$.

\section{Interruption Periods}

As the administration of interruption trials 2 and 3 depended on the successful mastery of trial 1 for each task, the absolute number of interruption periods varied across individuals, ranging from 3 to 12 interruptions per child in the autism group $(M=7.3)$ and 4 to 12 interruptions per child in the DD group $(M=10.8)$. To adjust for the different number of interruption periods between subjects, individual mean proportions were calculated for each measure (see below) that took into account the total number of interruption trials for each child. Importantly, all children mastered at least two tasks (trial 1) successfully and, therefore, all children could be included in the analyses of interruption periods. 
Behavior For each child, individual mean proportions were calculated (the number of behaviors, divided by the total number of 15 -second-interruption periods administered). This measure is presented in Fig. 3. Independentsample $t$-tests comparing the autism and the DD group revealed no significant group differences for Disengagement $(t(28)=0.92, p=0.18)$ or Individual Attempts $(t(28)=0.95, p=0.18)$. However, for Partner-Orientation, a significant group difference was found such that children with autism showed fewer behaviors that were oriented to the partner than children with developmental delay $(t(28)=-1.78, p<0.04)$.

Communicative Attempts Individual mean proportions (frequency of communicative attempts, divided by the total number of 15-second-interruption periods administered) were calculated for each type of communicative attempt. These measures are presented in Table 2. Independentsamples $t$-tests were conducted to compare each type of communicative attempt between groups. First, we analyzed all communicative attempts, proximal and distal, the children made and found no significant difference between groups $(t(28)=-0.81, p=0.21)$. In a second step, we analyzed different kinds of communicative attempts. Results revealed no significant group differences for proximal, requesting communicative attempts $(t(28)=0.12$, $p=0.45)$ or distal, requesting communicative attempts $(t(28)=-0.94, p=0.18)$. In a further step of analyses, we

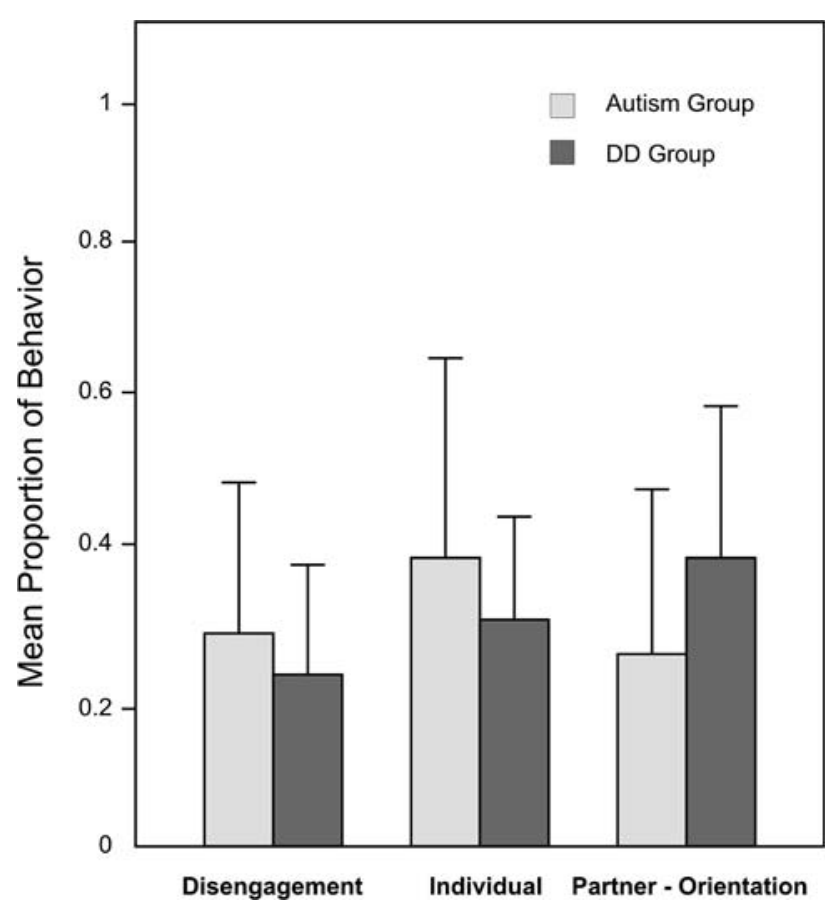

Fig. 3 Mean proportions and standard deviations of behavior during interruption periods as a function of group (Study 2) compared a subgroup of distal requestive communicative attempts (vocal or gestural) - with and without eye contact - between groups. Results indicated a significant group difference for distal requestive communicative attempts with eye contact $(t(28)=-1.88, p<0.04)-$ such that that children with autism made fewer. There was no difference for distal requestive communicative attempts without eye contact $(t(28)=0.89, p=0.19)$. To summarize, in those trials in which they were skillful enough at cooperation to be administered an interruption period, children with autism directed as many communicative attempts toward a nonresponding partner as did children with developmental delay, but they made fewer coordinated bids that involved eye contact with the partner in combination with vocal expression and/or point.

\section{Correlation with Helping Behaviors}

We correlated the difference between helping behaviors (mean proportion) in experimental condition and control condition from Study 1 as a measure of helping and the mean proportion of passed tasks from Study 2 as a measure of cooperation. Because of large proportions of tied observations we estimated $p$-values of correlation coefficients using an approximate permutation procedure (Software written by Roger Mundry) running 10,000 permutations. Spearman's rank correlations of helping and cooperative behaviors were calculated for both groups separately. They revealed a significant positive correlation for the autism group $(r=0.70, N=14, p<0.02)$ and a trend for a positive correlation in the DD group $(r=0.53$, $N=15, p=0.06)$.

\section{Discussion}

In terms of task performance, in three of the four cooperation tasks children with autism performed less successfully than children with developmental delay. When the adult ceased participating during the interruption periods, they engaged in less partner-directed behaviors than the children with developmental delay. However, in cases in which they attempted to re-engage the adult, the only difference among four different communicative behaviors examined involved poorer coordination of gaze with another communicative behavior.

It is unlikely that children with autism struggled with the tasks because they did not understand the properties of the apparatuses or had problems handling them. All four of the tasks were designed to be cognitively simple. Actions included pulling on a handle to separate the parts of a tube, pushing a cylinder up or grasping a toy from it, jiggling a 
Table 2 Frequencies of communicative attempts as a function of group (Study 2)

\begin{tabular}{llllll}
\hline Group & \multicolumn{5}{l}{ Communicative attempt } \\
\cline { 2 - 6 } & All & Proximal, requesting & Distal, requesting & Distal, requesting w/e.c. & Distal, requesting w/o e.c. \\
\hline Autism & $0.74(0.82)$ & $0.18(0.21)$ & $0.59(0.75)$ & $0.23(0.34)$ & $0.31(0.44)$ \\
DD & $1.04(1.15)$ & $0.17(0.22)$ & $0.89(1.00)$ & $0.67(0.83)$ & $0.19(0.28)$ \\
\hline
\end{tabular}

Note: Mean proportions (standard deviations in parentheses)

trampoline up and down, and putting a block down a chute or catching a block coming out of a chute. We do not believe that the difficulty arises from the individual actions the child must perform, or even from understanding the means-end relation by which the adult's action makes something happen with an object of interest. Several studies with typically developing children of a comparable mental age have shown that they can coordinate their actions with adults (Eckerman and Didow 1989; Eckerman and Stein 1990). More specifically, typically developing children at 18 months of age who were tested in almost identical tasks and procedures as used in the current study were able to coordinate their actions with those of another person, even when no verbal instructions or cues were provided (Warneken et al. 2006). The difficulties of children with autism in these cooperative tasks might thus reflect underlying autism-specific deficits.

The present findings contradict the results of Downs (2003) who found that 5- to 9-year-old children with autism cooperated as successfully as typically developing children in a Prisoner's Dilemma task. One possible explanation for this difference could be that the social skills of children with autism might improve over the developmental course as suggested by Sigman and Ruskin (1999). Downs discusses the appropriateness of the task as it did not involve direct cooperative interaction and behavioral coordination as in the current study. Results of the present study are in line with the findings by Jahr et al. (2000) who also found that children with autism performed poorly in cooperative game tasks. Interestingly, Jahr et al. showed that children's performance improved after a language-based intervention. The present study had a different focus - namely, to explore cooperation in younger children with autism. The rationale for this focus derived from the study by Warneken et al. (2006) who found that 18-month-old typically developing infants are able to master these tasks independent from language. However, as the older children in the study by Jahr et al. seemed to benefit from the intervention, it would be helpful to consider children's verbal skills and the effects of training on their cooperative performance in these nonverbal tasks in future research.

In the present study, the group differences regarding task performance were very clear. Fewer children with autism were successful compared to the children with developmental delay in three of four tasks. Also evident were group differences during the interruption periods: children in the autism group produced fewer partner-oriented behaviors, and they used few distal communicative attempts in which they established eye contact with the partner. This might indicate an impaired understanding of the partner's role within the cooperative dyad (Tomasello et al. 2005). However, efforts to re-engage a partner also require social initiative and the capacity to use intentional gestures to communicate imperative goals. These are other areas of known impairments in autism and are in fact key symptoms sampled during behavioral assessments of autism in tools like the Autism Diagnostic Observation Schedule (Lord et al. 1999). Importantly, it has to be noted that the groups differed only regarding communicative attempts that involve eye contact. Overall, the children with autism made as many communicative attempts to re-engage the adult as the children with developmental delay. This might suggest that children with autism use other ways to express their communicative intentions.

We should note that although the tasks were mainly nonverbal (and had a visual demonstration), there were some gestures and speech used by the adult as bids for cooperation. Since our groups were not matched on language level, the group differences may be attributable to some unknown degree to autism-specific difficulties in the comprehension of communicative bids for engagement. On the other hand, some of the ceiling effects in the DD group make it possible that some group differences are even more marked than is apparent from the analyses.

In sum, cooperation with others might represent a unique form of social engagement with its own unique components - shared goals, shared attention, and shared plans of action (intentions). It would certainly seem to be a worthy goal to attempt to identify which of these components cause problems for children with autism, so that one could begin to find ways to assist them in developing this fundamental social capacity. In this respect, the Jahr et al. (2000) article provides an interesting intervention. These researchers demonstrated that a combination of observation and verbal restatement of a cooperative activity improved the ability of children with autism to participate in similar cooperative play activities. Observation alone was not 
sufficient; the verbal rehearsal was an important part of the intervention, perhaps by engaging both procedural and declarative learning processes, or by creating a multimodal learning opportunity. The combined findings from the present and past descriptive studies of cooperation in autism and this intervention study strongly suggest that children with autism are capable of developing greater awareness of others and a greater sense of shared goals and shared intentions when there are environmental supports, scaffolds for such experiences, and practice. The fact that Jahr et al. (2000) found that cooperative performance generalized across situations and people strongly suggests that children with autism experience intrinsic rewards from such experiences. Findings suggest that targeted learning activities that emphasize helping and cooperative activities should be a part of social interventions for children with autism.

\section{General Discussion}

Taken together, the current studies paint an interesting picture of skills, weaknesses, and motivations for social behaviors in children with autism. As seen in these studies, young children with autism demonstrated skills and motivation to help others, at least in very simple situations such as someone reaching for something unsuccessfully, as well as some skills in more complex cooperative activities. As in studies of other social behaviors in autism, there are some significant group differences in performance and other areas in which there are no autism-specific differences. The social impairment is not an absence of social behavior, but rather decrements in certain specific types of social behavior (Rogers and Pennington 1991). Social abilities in autism demonstrate patterns of partial accomplishments and some capacity for social engagement.

According to the evolutionary account proposed by Tomasello et al. (2005), the ability to engage in cooperative activities with shared goals, shared intentions, and shared attention may be unique to humans. From an ontogenetic perspective, the ability to share intentions and attention develops as two developmental trajectories intertwine during the first two years of life: infants develop the capacity to understand other persons' goals and intentions as well as the skills and motivation to share goals and intentions with others. The present studies provide further support for this general proposal. We found that children with autism assisted the adult in the helping tasks, which involved recognizing another person's individual goals and the motivation to assist. However, the cooperation tasks were more challenging for them. They appear to be more complex than the helping tasks because they involve two people coordinating their behavior for a common goal. One hypothesis is that children with autism at this age and developmental level have great difficulty forming shared goals with others that involve joint intentions (plans) and joint attention/communication. The fact that children with autism did not very frequently attempt to re-engage the adult when she refrained from the interaction is consistent with this hypothesis - although this may also reflect core difficulties with communication or initiative as discussed above. We found this impairment even though this kind of regulatory communication is easier for children with autism than other types of nonverbal communication, and has been found to be unimpaired in some studies (Mundy et al. 1986). Despite the differences with typically developing children and children with developmental delay and in contrast to the proposal by Tomasello et al. (2005), it is noteworthy that, overall, the majority of the children with autism performed the cooperative tasks.

Another potential hypothesis to explain the poor performance of the children with autism in the cooperation study concerns imitation. Children observed two adults cooperating in these novel tasks before they engaged in the tasks themselves. The reliance on observational learning taps an area of impairment in autism, as documented by a host of imitation studies (see Rogers and Williams 2006, for a recent review). Furthermore, imitating novel actions and action sequences have recently been documented to be specifically impaired in early autism (Rogers et al. 2003). Understanding of pantomime and gesture are additional affected areas in early autism (Rogers et al. 1996). Thus, the means-end relations in the tasks were well within the cognitive abilities of the group with autism, and there was no requirement for understanding speech. However, modeling and gesture present other unique challenges to young children with autism, challenges that may well be inextricably connected to difficulties with sharing goals, the focus of this study.

Finally, in terms of motivation, we should note that we designed the tasks as attractive and fun for the children as possible. In some cases, small items of food were used as the object to retrieve or as reinforcement. However, another possible hypothesis could be that motivational deficits rather than deficits in social skills underlie the poorer performance of the children with autism. Theories that suggest autism involves a different developmental course set in motion by early differences in starting states, either involving the salience of social stimuli (Dawson et al. 2004), or the ability to mirror or imitate others' actions (Rogers and Pennington 1991) would predict problems with such tasks due to downstream effects of earlier social deficits. We are not able to answer the question to what extend motivational deficits account for poorer 
performance of the children with autism. In addition, it may be important that in the tasks of the current study the goal and roles were predetermined. It would be interesting to study cooperative behavior in less constrained contexts, when adult and child have to choose among a variety of tasks and negotiate roles, and then to compare these data with the present study in which the adult chooses the cooperative task and the roles are set.

Our study presents several limitations. Given that these are newly developed tasks used with small sample sizes, independent replication of the findings is needed before we can assess the generalizability of the findings. As argued above, we should also note that although the tasks were mainly nonverbal, there were some gestures and speech used by the adult. As our groups were not matched on language level, the group differences may partially be explained by some unknown degree to autism-specific difficulties in the comprehension of communicative bids for engagement. Finally, due to practical constraints we were not able to include individual information regarding interventions that the children received. It would be interesting to see if there were correlations with the kind and amount of interventions these children receive. We are not at the moment prepared to choose among these various explanatory possibilities.

The present studies obviously represent only a first step in determining the skills and motivations that children with autism have for interacting with others in helping and cooperative behaviors. These social behaviors are very complex in several ways: the number of actions involved, the motivational state, the temporal flow regarding sequencing of steps, and coordinating actions between two people. Yet, joint actions involved in social cooperation may be the foundational experiences for developing symbolic representations of social events - the elements of social cognition which are impaired in autism. Thus, examination of the social interactions during these cooperative exchanges is likely to provide unique information about one source of social-cognitive impairment in autism. It also provides a paradigm for examining individual differences in early social development, motivation, and awareness in young children with autism. Finally, these and similar activities offer a potential frame for interventions to enhance social development in children with autism.

Acknowledgments These studies were conducted through a collaboration of the Max Planck Institute for Evolutionary Anthropology, Leipzig, Germany, and the University of California, Davis, M.I.N.D. Institute, Sacramento, CA, USA. Dr. Rogers and Ms. Colombi's time were partially supported by funding from the National Institute of Child Health and Human Development U19 HD35468, and a Fulbright scholarship. Many thanks to the participating children and their families. Thanks also to Petra Jahn for technical support, and to Daniel Stahl and Roger Mundry for statistical advice.

\section{Appendix 1: Detailed Description of Procedure for Each Task (Study 2)}

Tube-with-handles Task

\section{Demonstration}

E1 showed a toy to the child and then put it into the tube. E1 and E2 closed the tube, saying "close!" and put it on the floor. In an exaggerated way, they then picked up one handle each and opened the tube by pulling simultaneously, saying "we open!". They displayed positive affect after opening the tube. After the demonstration, another toy was put inside and the tube was closed.

\section{Trial 1}

E1 held one handle, ready to pull, and encouraged the child to join nonverbally by alternating gaze between the child and the tube, and verbally by saying "open!". If the child was not successful (successful performance: retrieving the toy) after 60 seconds, a second and, if needed, third demonstration were given. Between trials, another toy was shown to the child and put into the tube, and the tube was closed by E1 and E2.

\section{Trials 2 and 3}

E1 encouraged the child to engage as in trial 1. After E1 and child had started pulling simultaneously, E1 suddenly put the tube on the floor and released the handle. After 15 seconds, she picked up the handle again and engaged in pulling.

\section{Elevator Task}

\section{Demonstration}

One experimenter pushed the cylinder, baited with two toys, up, pointed to the object with positive affect and gently dropped the cylinder. The other experimenter was positioned on the retrieval side of the box and pointed to the toys when accessible as the cylinder was pushed up, displaying positive affect. She did not take one of the two toys until the last of three repetitions. One toy remained inside the cylinder for trial 1 .

\section{Trial 1}

If the child was in role A (retrieve), E1 pushed the cylinder up and encouraged the child to join nonverbally by pointing toward the toy and alternating gaze between the child 
and the toy, and verbally by saying "the toy!". If the child was in role $B$ (push), E1, positioned on the retrieval side, made a grasping gesture toward the cylinder, alternated gaze between the child and the cylinder, and said "the toy!". If the child was not successful (successful performance: retrieval of the toy) after 60 seconds, a second and, if needed, third demonstration were given. Between trials, the cylinder was loaded with another toy, invisibly to the child.

\section{Trials 2 and 3}

After E1 and the child had cooperatively engaged in the task, an interruption period followed. If child was in role $A$, E1 gently dropped the cylinder. If the child was in role $B$, E1 withdrew her hand from grasping the toy and put it on her knees. After 15 seconds, she started to push again or made the grasping-gesture, respectively.

Trampoline Task

\section{Demonstration}

E1 and E2 grabbed the rim of the trampoline and made the block bounce in an exaggerated manner, showing positive affect.

\section{Trial 1}

E1 picked up the trampoline with the block on top and encouraged the child to join nonverbally by alternating gaze between the child and the trampoline, and verbally by saying: "bounce!" If the child did not succeed (successful performance: joint play for a total of 5 seconds), a second and, if needed, third demonstration were administered.

\section{Trials 2 and 3}

After E1 and the child had started to engage in joint play, E1 put the trampoline on the floor and released the rim. After 15 seconds of interruption, she picked up the trampoline again and resumed playing.

Double-tubes Task

\section{Demonstration}

One experimenter, positioned at the upper end of the tubes, threw the block into the black tube. The other experimenter caught the block with the can at the opposite side of the apparatus. Both experimenters displayed positive affect after the block went into the can. After one repetition, the experimenters switched tubes in an exaggerated manner and the procedure was repeated with the white tube two more times.

\section{Trial 1}

If the child was in role $A$ (throw), E1 held the can underneath the lower end of the white tube, encouraging the child to join nonverbally by placing the block on the apparatus and alternating gaze between the child and the apparatus and verbally by saying "play!". If the child was in role $B$ (catch), E1 held the block in the position ready to throw at the upper end of the white tube, encouraging the child to join by gaze alternation and saying "play!". If the child was not successful after 60 seconds (successful performance: child chooses same tube as adult at least once for each tube), a second and, if needed, third demonstration were administered.

\section{Trials 2 and 3}

If the child was in role A, E1 withdrew the can holding it upright in front of her body. If child was in role B, E1 withdrew the block holding it upright in front of her body. After 15 seconds, E1 resumed playing by holding the can underneath the tube or indicating to throw the block into the tube again, respectively.

\section{Appendix 2: Coding Schema for Interruption Periods (Behavior, Communicative Attempts) (Study 2)}

\begin{tabular}{l}
\hline Category \\
\hline Overall behavior (majority of time) \\
Disengagement \\
$\begin{array}{l}\text { Child leaves apparatus or plays without } \\
\text { pursuing the goal of the task like } \\
\text { banging on the apparatus, climbing on } \\
\text { it, repetitive behaviors, etc. } \\
\text { Child attempts to retrieve the object } \\
\text { individually or attempts to continue the } \\
\text { game herself (e.g., in the elevator task, } \\
\text { the child would come over to the } \\
\text { experimenter's side and push the } \\
\text { cylinder up herself while reaching for } \\
\text { the object; in the tube-with-handles } \\
\text { task, the child tries to hold both } \\
\text { handles, or peel it open on one side) } \\
\text { Child remains on correct side of the } \\
\text { apparatus and is ready to perform her } \\
\text { role (waiting); or child is focused on } \\
\text { E1 and insistently tries to re-engage her } \\
\text { (e.g., by pushing the cylinder of the } \\
\text { elevator up, pointing at the object, } \\
\text { and vocalizing while looking at E1) } \\
\text { (re-engagement) }\end{array}$ \\
Partner-orientation $\quad$
\end{tabular}


continued

Category

Definition

Communicative attempt: proximal requesting

Placing or touching Child either moves the apparatus towards E1 or pushes E1/her hand towards the apparatus

\section{Communicative attempt: distal requesting with eye contact}

Eye contact and pointing Child establishes eye contact with E1 and and/or vocalizing vocalizes or points to the apparatus, or a combination of all three

Communicative attempt: distal requesting without eye contact

Verbal and/or point Child makes clear verbal utterance and/or points to the apparatus

\section{References}

Aldridge, M. A., Stone, K. R., Sweeney, M. H., \& Bower, T. G. R. (2000). Preverbal children with autism understand the intentions of others. Developmental Science, 3(3), 294-301.

American Psychiatric Association (1994). Diagnostic and statistical manual of mental disorders (4th ed.). Washington, DC: APA.

Ashley, J., \& Tomasello, M. (1998). Cooperative problem-solving and teaching in preschoolers. Social Development, 7(2), 143-163.

Baron-Cohen, S. (1989a). Perceptual role taking and protodeclarative pointing in autism. British Journal of Developmental Psychology, 7(2), 113-127.

Baron-Cohen, S. (1989b). The theory of mind hypothesis of autism: A reply to Boucher. British Journal of Disorders of Communication, 24, 199-200.

Baron-Cohen, S. (1995). Mindblindness: An essay on autism and theory of mind. Cambridge: MIT Press/Bradford Books.

Bono, M. A., Daley, T., \& Sigman, M. (2004). Relations among joint attention, amount of intervention and language gain in autism. Journal of Autism and Developmental Disorders, 34(5), 495-505.

Bratman, M. E. (1992). Shared cooperative activity. The Philosophical Review, 101(2), 327-341.

Brownell, C. A., \& Carriger, M. S. (1990). Changes in cooperation and self-other differentiation during the second year. Child Development, 61(4), 1164-1174.

Brownell, C. A., \& Carriger, M. S. (1991). Collaborations among toddler peers: Individual contributions to social contexts. In L. B. Resnick, J. M. Levine, \& S. D. Teasley (Eds.), Socially shared cognition (pp. 365- 383). Washington, DC: American Psychological Association.

Capps, L., Sigman, M., \& Mundy, P. (1994). Attachment security in children with autism. Development and Psychopathology, 6(2), 249-261.

Carpenter, M., Pennington, B. F., \& Rogers, S. J. (2001). Understanding of others' intentions in children with autism. Journal of Autism and Developmental Disorders, 31(6), 589-599.

Charman, T., Swettenham, J., Baron-Cohen, S., Cox, A., Baird, G., \& Drew, A. (1997). Infants with autism: An investigation of empathy, pretend play, joint attention, and imitation. Developmental Psychology, 33(5), 781-789.

Cohen, J. (1960). A coefficient of agreement for nominal scales. Educational and Psychological Measurement, 10(1), 37-46.

Dawson, G., Toth, K., Abbott, R., Osterling, J., Munson, J., Estes, A., \& Liaw, J. (2004). Early social impairments in autism: Social orienting, joint attention, and attention to distress. Developmental Psychology, 40(2), 271-283.

Downs, M. (2003). Cooperation in high functioning children with autism. Ann Arbor, MI: UMI Dissertation Services.

Eckerman, C. O., \& Didow, S. M. (1989). Toddlers' social coordinations: Changing responses to another's invitation to play. Developmental Psychology, 32(1), 141-152.

Eckerman, C. O., \& Stein, M. R. (1990). How imitation begets imitation and toddlers' generation of games. Developmental Psychology, 26(3), 370-378.

Eisenberg, N., \& Fabes, R. A. (1998). Prosocial development. In W. Damon (Series Ed.) \& N. Eisenberg (Vol. Ed.), Handbook of child psychology: Vol. 3. Social, emotional, and personality development (5th ed., pp. 701-778). New York: Wiley.

Jahr, E., Eldevik, S., \& Eikeseth, S. (2000). Teaching children with autism to initiate and sustain cooperative play. Research in Developmental Disabilities, 21, 151-169.

Kasari, C., Sigman, M., Mundy, \& Yirmiya (1990). Affective sharing in the context of joint attention interactions of normal, autistic and mentally retarded children. Journal of Autism and Developmental Disorders 20(1), 87-100.

Kasari, C., Sigman, M., \& Yirmiya, N. (1993). Focused and social attention of autistic children in interactions with familiar and unfamiliar adults: A comparison of autistic, mentally retarded, and normal children. Development and Psychopathology, 5, 403-414.

Kuhlmeier, V. A., Wynn, K., \& Bloom, P. (2003). Attribution of dispositional states by 12-month-olds. Psychological Science, 14(5), 402-408.

Leekam, S., Baron-Cohen, S., Perrett, D., Milders, M., \& Brown, S. (1997). Eye-direction detection: A dissociation between geometric and joint attention skills in autism. British Journal of Developmental Psychology, 15, 77-95.

Leekam, S. R., Lopez, B., \& Moore, C. (2000). Attention and joint attention in preschool children with autism. Developmental Psychology, 36(2), 261-273.

Liszkowski, U., Carpenter, M., Striano, T., \& Tomasello, M. (2006). Twelve- and 18-month-olds point to provide information for others. Journal of Cognition and Development, 7(2), 173-187.

Lord, C., Rutter, M., DiLavore, P., \& Risi, S. (1999). Autism diagnostic observation schedule, Generic (ADOS-G). Los Angeles: WPS.

Meltzoff, A. (1990). Foundations for developing a concept of self: The role of imitation in relating self to other and the value of social mirroring, social modeling, and self practice in infancy. In Cicchetti, D., \& Beeghly (Eds.), The MacArthur foundation series on mental health and development (pp. 139-164). Chicago, IL, USA: University of Chicago Press (Viii, 399 pp.).

Meltzoff, A. (1995). Understanding the intentions of others: Re-enactment of intended acts by 18 -month-old children. Developmental Psychology, 31(5), 838-850.

Mullen, E. M. (1995). Mullen scales of early learning. Circle Pines, MN: American Guidance Service.

Mundy, P., Sigman, M., \& Kasari, C. (1993). The theory of mind and joint attention deficits in autism. In S. Baron-Cohen, H. TagerFlusberg, \& D. Cohen (Eds.), Understanding other minds: Perspective from autism (pp. 181-203). Oxford, UK: Oxford University Press.

Mundy, P., Sigman, M., Ungerer, J. A., \& Sherman, T. (1986). Defining the social deficits in autism: The contribution of nonverbal communication measures. Journal of Child Psychology and Psychiatry and Allied Disciplines, 27(5), 657-669.

Mundy, P., \& Willoughby, J. (1996). Nonverbal communication, joint attention, and early socioemotional development. In M. Lewis, \& M. W. Sullivan (Eds.), Emotional development in atypical 
children (pp. 65-87). Hillsdale, NJ: Lawrence Erlbaum Associates, Inc.

Richerson, P., \& Boyd, R. (2005). Not by genes alone: How culture transformed human evolution. Chicago, IL: University of Chicago Press.

Rogers, S. J. (1999). An examination of the imitation deficit in autism. In J. Nadel, \& G. Butterworth (Eds.), Imitation in infancy (pp. 254-283). Cambridge, UK: Cambridge University Press.

Rogers, S. J., Bennetto, L., McEvoy, R., \& Pennington, B. F. (1996). Imitation and pantomime in high-functioning adolescents with autism spectrum disorders. Child Development, 67(5), 2060-2073.

Rogers, S. J., Hepburn, S. L., Stackhouse, T., \& Wehner, E. (2003). Imitation performance in toddlers with autism and those with other developmental disorders. Journal of Child Psychology and Psychiatry and Allied Disciplines, 44(5), 763-781.

Rogers, S., Ozonoff, S., \& Maslin-Cole, C. (1993). Developmental aspects of attachment behavior in young children with pervasive developmental disorders. Journal of the American Academy of Child \& Adolescent Psychiatry, 32(6), 1274-1282.

Rogers, S. J., \& Pennington, B. F. (1991). A theoretical approach to the deficits in infantile autism. Development and Psychopathology, 3(2), 137-162.

Rogers, S. J., \& Williams, J. H. G. (2006). Imitation and the social mind: Autism and typical development. New York, NY, US: Guilford Press.

Ross, H. S., \& Lollis, S. P. (1987). Communication within infant social games. Developmental Psychology, 23(2), 241-248.

Sigman, M., \& Mundy, P. (1989). Social attachments in autistic children. Journal of the American Academy of Child \& Adolescent Psychiatry, 28(1), 74-81.

Sigman, M., Mundy, P., Sherman, T., \& Ungerer, J. A. (1986). Social interaction of autistic, mentally retarded, and normal children with their caregivers. Journal of Child Psychology and Psychiatry and Allied Disciplines, 27(5), 647-656.
Sigman, M., \& Ruskin, E. (1999). Continuity and change in the social competence of children with autism, down syndrome, and developmental delays. Monographs of the Society for Research in Child Development, 64(1), v-114.

Sigman, M., \& Ungerer, J. (1984). Cognitive and language skills in autistic, mentally retarded, and normal children. Developmental Psychology, 20(2), 293-302.

Smith, I. M., \& Bryson, S. E. (1998). Gesture imitation in autism I: Nonsymbolic postures and sequences. Cognitive Neuropsychology, 15(6-8), 747-770.

Sprent, P., \& Smeeton, N. C. (2001). Applied nonparametric statistical methods (3rd ed.). London: Chapman and Hall/CRC.

Stern, D. N. (1985). The interpersonal world of the human infant. New York: Basic Books.

Stone, W. L., Ousley, O. Y., \& Littleford, C. D. (1997). Motor imitation in young children with autism: What's the object? Journal of Abnormal Child Psychology, 25(6), 475-485.

Tomasello, M., Carpenter, M., Behne, T., \& Moll, H. (2005). Understanding and sharing intentions: The ontogeny and phylogeny of cultural cognition. Behavioral and Brain Sciences, 28(5), 675-691.

Warneken, F., Chen, F., \& Tomasello, M. (2006). Cooperative activities in young children and chimpanzees. Child Development, 77(3), 640-663.

Warneken, F., \& Tomasello, M. (2006). Altruistic helping in human infants and young chimpanzees. Science, 311(3), 1301-1303.

Williams, J. H. G., Whiten, A., \& Singh, T. (2004). A systematic review of action imitation in autistic spectrum disorder. Journal of Autism and Developmental Disorders, 34(3), 285-299.

Yirmiya, N., Kasari, C., Sigman, M., \& Mundy, P. (1989). Facial expressions of affect in autistic, mentally retarded and normal children. Journal of Child Psychology and Psychiatry and Allied Disciplines, 30(5), 725-735. 